\title{
Considerations on the DEMO pellet fuelling system
}

\author{
P. T. Lang ${ }^{\mathrm{a}}$, Ch. Day ${ }^{\mathrm{b}}$, E. Fable ${ }^{\mathrm{a}}$, Y. Igitkhanov ${ }^{\mathrm{b}}$, F. Köchl ${ }^{\mathrm{c}}$, R. Mooney ${ }^{\mathrm{d}}$, B. Pegourie ${ }^{\mathrm{e}}$, \\ B. Ploeckl ${ }^{\mathrm{a}}$, R. Wenninger ${ }^{\mathrm{a}, \mathrm{f}}, \mathrm{H}$. Zohm ${ }^{\mathrm{a}}$ \\ ${ }^{a}$ Max-Planck-Institut für Plasmaphysik, Boltzmannstr. 2, 85748 Garching, Germany \\ ${ }^{b}$ Karlsruhe Institute of Technology, 76021 Karlsruhe, Germany \\ ${ }^{c}$ Association EURATOM-Ö AW/ATI, Atominstitut, TU Wien, 1020 Vienna, Austria \\ ${ }^{d}$ Culham Centre for Fusion Energy, Culham Science Centre, Oxfordshire, OX14 3DB, UK \\ ${ }^{e}$ CEA, IRFM, 13108 Saint-Paul-lez-Durance, France \\ ${ }^{f}$ EFDA, Garching, Germany
}

\begin{abstract}
The Demonstration Fusion Power Reactor DEMO is the step foreseen to bridge the gap between ITER and the first commercial fusion power plant. One key element in the European work plan for DEMO is the elaboration of a conceptual design for a suitable core particle fuelling system. First considerations for such a system are presented in this contribution. Following the well-considered ITER solution, most analysis performed in this study assumes conventional pellet technology will be used for the fuelling system. However, taking advantage of the less compressed time frame for the DEMO project, several other techniques thought to bear potential for advanced fuelling performance are considered as well. In a first, basic analysis all actuation parameters at hand and their implications on the fuelling performance were considered. Tentative transport modelling of a reference scenario strongly indicates only particles deposited inside the plasma pedestal allow for efficient fuelling. Shallow edge fuelling results in an unbearable burden on the fuel cycle. Sufficiently deep particle deposition seems technically achievable, provided pellets are launched from the torus inboard at sufficient speed. All components required for a DEMO pellet system capable for high speed inboard pellet launch are already available or can be developed in due time with reasonable efforts. Furthermore, steps to integrate this solution into the EU DEMO model are taken.
\end{abstract}

Keywords: Tokamak, Pellet fuelling, Launcher technology, DEMO

\section{Introduction}

DEMO is a machine that should bridge the gap between ITER and the first commercial fusion power plant. Crucial elements for many DEMO designs are net electricity production and T-self-sufficiency as well as reasonable reliability and availability. Hence, DEMO requires significant progress compared with ITER on the so-called 'Demo Physics Issues', defined as issues that do not have to be resolved for ITER to achieve its mission, but are critical for DEMO [1]. Some will have large implications on the requirements for the core particle fuelling system, e.g. with respect to steady state operation, high density operation and exhaust with high heat flux densities. Consequently, the work plan for the implementation of the EFDA roadmap to the realization of fusion energy [2] assigns as one key deliverable "The conceptual design description of the DEMO fuelling system including the selection and further development of fuelling technologies to satisfy the requirements". Technology requirements have to be derived taking into account the demands and boundary conditions imposed by the according target plasma scenarios able to provide the required Deuterium (D) - Tritium (T) fuel mixture to maintain the plasma as foreseen by the plasma scenarios.

DEMO will be different to ITER; hence additional requests will apply for the core fuelling system. Nevertheless, it is worthwhile to take a closer look at the ITER solution selected for core particle fuelling. ITER is the largest experiment devoted to fusion oriented research and technology and will have faced already many of the problems expected to be encountered by DEMO. The next step is to evaluate ITER's solution against DEMO requirements. To check if a scale up is possible or which elements of the ITER solution can be used for DEMO, if not entirely, at least in parts.

For DEMO, it is also possible to take advantage of the longer time span still ahead for preparations and R\&D. While some decisions taken by ITER had been forced by the urgent need to make solutions and start to design and build the system, resulting ITER solutions can be reconsidered for DEMO. Making the step from ITER to DEMO hence allows integrating more advanced technology and physics. For the task of core particle fuelling, ITER disregarded fuelling by conventional gas bleeding (due to the saturation of the edge density) and neutral beam injection (for the insufficient total particle flux); this will hold for DEMO most probably as well [3]. Some advanced fuelling techniques like the injection of compact tori (CT) were seriously considered [4] but it was found that operational parameters were not yet up to reasonable requirements at the time the decision had to be taken. On the basis of the longer DEMO time scale it seems worthwhile to renew such attempts in a broader approach. We revisited status and potential of all suitable advanced fuelling technologies as possible systems for core matter fuelling.

For ITER core particle fuelling, a pellet launching system basing on conventional technology was chosen [5]. It is expected to launch the fuelling pellets from the torus inboard, granting vessel access via transfer tubes 
installed below the divertor structure. The resulting complex injection track will limit the launch speed to $300 \mathrm{~m} / \mathrm{s}$, as found during investigations in mock up test facility. Expectedly this will just meet fuelling requirements [3], leaving only very little headroom for maneuvering to optimize the performance. Despite this limitation, the ITER system will nevertheless act as a ground-breaking endeavor. It is expected to demonstrate several achievements required for DEMO for the first time. It fosters technology needed for core fuelling on a reactor grade level, especially with respect to steady state operation and working with $\mathrm{T}$. Consequently, for DEMO a baseline solution for the core matter injection system will rely only on conventional pellet technology. Hence, detailed considerations made in the following paper will be based on the assumption pellets are chosen for the fuelling tasks. Evidently, such a system would have also the potential to serve for other tasks like e.g. ELM control or disruption handling; however according implications have not yet been regarded.

Advanced inboard pellet fuelling [6] will be required for DEMO. Taking advantage of the gain, due to the underlying physics, this requirement dictates the application of pellet guiding technology. The predicted $300 \mathrm{~m} / \mathrm{s}$ pellet speed solution for ITER is marginal for DEMO. DEMO has to go for a different, more ambitious approach. Hence, a first and most urgent task was to find out if higher speed would be possible and to what extent this could improve fuelling behavior. As it will be shown in this paper, it seems beneficial to aim for a pellet speed of about $1000 \mathrm{~m} / \mathrm{s}$. When considering the available technology, indications are that an appropriate technical solution is viable. In fact a first request has already been made to integrate this system into the European DEMO reference design, details shown also in this paper.

Although we follow initially the route paved by ITER and other similar conventional systems, considerations for alternative solutions are made as well. The alternatives are reviewed to find out if more detailed investigations should be carried out. Several candidate technologies have been identified so far, to be found at the end of this paper. The shortlist represents the current status only; further techniques might still be added for further reviewing and validation.

\section{Actuation parameters of the pellet system and fuelling performance}

When a pellet system has to be laid out for a specific task, several parameters can be adjusted. These actuation parameters are:

- Pellet mass [atoms]

- Pellet speed $[\mathrm{m} / \mathrm{s}]$

- Pellet rate $[\mathrm{Hz}]$ (times the pellet size this determines the pellet particle flux [at/s])

- Pellet launching location/geometry

- Pellet material

To evaluate the impact of actuation parameters their influence on the fuelling performance has to be investigated. The fuelling performance in turn determines the required pellet particle flux to be applied in order to achieve the required fuelling. Consequently, a fuelling efficiency study has to be performed for any pellet parameter set, aiming for an overall optimized set. Once steady state is achieved, maximum fuelling efficiency is achieved with optimized single pellet efficiency. For our considerations, we assume the following actuation parameters:

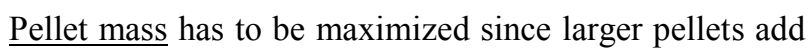
more particles to the inward part of the ablation profile. Deeper deposition improves the fuelling efficiency. However, pellets also cool down the plasma and can trigger instabilities. Most likely, there will be a pellet mass limit due to stability restrictions. Accordingly stability limit investigations for various DEMO scenarios have to be undertaken. Since no definite mass value can be provided at present, for a first estimate we adopted those values used by ITER.

Pellet speed has to be maximized since this also results in deeper penetration and particle deposition. Again, regions where instabilities can be triggered must be avoided. A maximum launch speed has to be defined to achieve maximum fuelling performance. As will be detailed later, this turns out to be a key challenge for the launching and transfer technology but also the entire design integration. Furthermore, there is a strong correlation between launch speed and location.

Pellet rate will depend on the single pellet efficiency. Once the efficiency is optimized this actuation parameters should be minimized to a value just able to fulfil the required fuelling task.

Pellet launch location is preferred from the torus inboard. Thus, plasmoid drift and pellet motion are in parallel direction. Precooling effects by drifting clouds and pellet acceleration by asymmetric heat flux shielding become strongest. Tilting of the launch path results in a reduced performance in case all other parameters are kept. However, as will be shown later in detail, the launch location has a great impact on the guiding system geometry and hence also on the path tilting angle and the maximum possible pellet transfer speed. Optimization is a compromise of all these boundary conditions and finding the maximum overall performance.

Pellet material for core fuelling has obviously to be the correct fuel mixture. To replenish burn up, $\mathrm{T}$ has to be delivered. As well, D losses must be substituted. Hence, a DT mixture tailored to keep the requested ratio in plasma core [7] is needed. The resulting value for the needed pellet D-T ratio is subject to detailed modelling.

In this context, considerations for possible novel techniques are envisaged. It is expected ITER, and DEMO in particular, will have to inject heavier gases like Neon, Argon or even Krypton for radiation buffering, and perhaps other gases like Nitrogen for performance improvement. In order to handle the throughput of such material with high efficiency, keeping the burden on the pumping system and the fuelling reprocessing facilities bearable, a pellet based 
option could turn out beneficial. It is also thought such gases, admixed to the fuel, could result in higher pellet stability. Enhanced stability could allow for higher maximum transfer speed within the prescribed transfer geometry. Moreover, the ablating material containing higher $\mathrm{Z}$ components will result in extra cooling of the ablating cloud surrounding the pellet, reducing the ablation rate and hence resulting in deeper pellet perturbation. Altogether, synergetic effects of admixed fuel could yield the potential to improve both fuelling performance and efficiency of buffering gas applications. According investigations just started [8].

\section{Pellet fuelling in DEMO: a first tentative modelling assessment}

Although many choices for a distinct plasma scenario are still pending, a first tentative modelling assessment of pellet core fuelling was performed. Its main intention was to find out the impact any efforts to increase the pellet speed would have. In particular to find out if a higher but still technical reasonable speeds compared to the ITER case could result in a significant efficiency enhancement. In a first step, the influence of the radial location of particle source was investigated. For this modelling, the ASTRA transport code [9] was employed. A DEMO1noCD scenario has been selected that is similar to the one described in [10]. For the code runs, a steady state particle source was implemented localized with a radial extension of $3.15 \mathrm{~cm}$ (Gaussian shape, FWHM). The magnitude of the source flux was then increased until the requested scenario density at the pedestal top ( 0.85 times the Greenwald density) was matched. The result, requested particle flux versus deposition location, is displayed in figure 1. For comparison, pellet rates are given using ITER size pellets.

It clearly shows the positive effect of deeper particle deposition, at least in the pedestal region. Particles deposited closer to the separatrix show short residual times and accordingly a high flux is needed. Beyond pedestal top (indicated by the dashed line) better particle confinement requires less flux. It should be noted the analysis was performed assuming steady state conditions of plasma transport. Repeated transient collapses of edge transport barrier observed during ELMs were disregarded. The effect of ELMs will make the particle deposition in the pedestal even less efficient, in particular if the ELM instability is triggered by the pellets. The basic result from this first analysis is that only particle deposition beyond the pedestal top will result in efficient core fuelling.

In a second step this information had to be transferred to suitable actuation parameters. Consequently ablation and deposition of the pellets had to be modelled. Choosing again the DEMO1noCD scenario, pellet ablation and particle deposition profile were calculated employing the JETTO code [11]. For the pellet parameters we took a mass of $6 * 10^{21} \mathrm{D}$ and three different pellets speeds: $300 \mathrm{~m} / \mathrm{s}, 1000 \mathrm{~m} / \mathrm{s}$ and $3000 \mathrm{~m} / \mathrm{s}$. As will be discussed later, the different speeds represent distinct technical solutions. Results are presented in figure 2 .

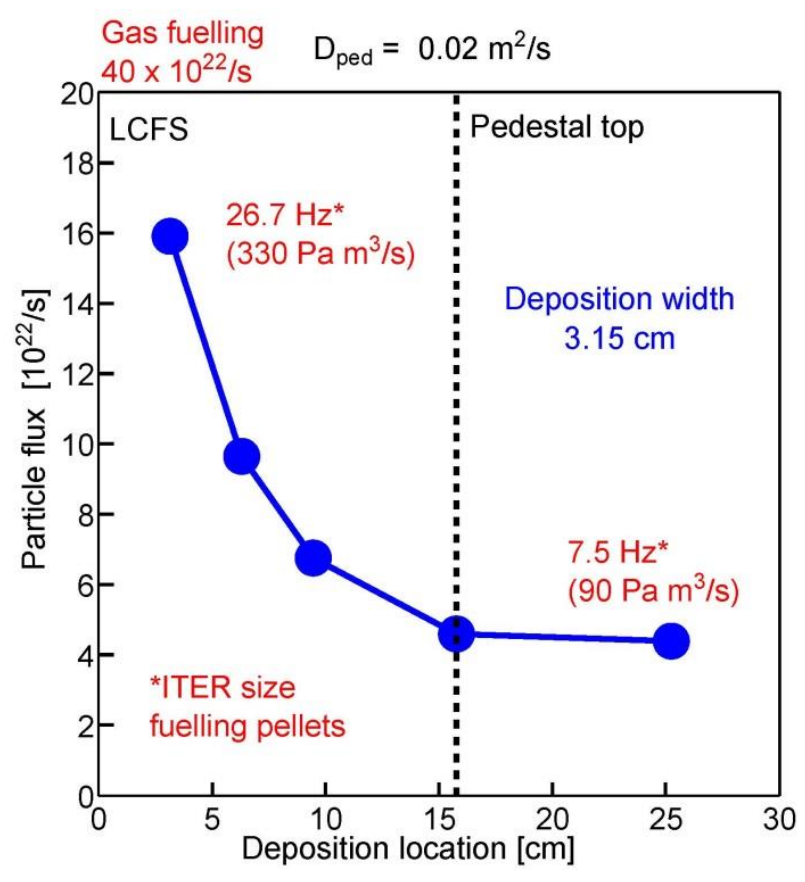

Fig. 1. Modelled particle flux required to establish targeted pedestal density in the DEMO1noCD scenario for a localized steady state particle source (extension $3.15 \mathrm{~cm}$ ) at different radial positions. Evidently, particle deposition deeper into the pedestal results in a better fuelling performance. Beyond the pedestal, a stagnation of this performance takes place.

Dashed lines display ablation rates calculated without drift effects. In any case almost all particles are ablated essentially in the pedestal region (pedestal top is indicated). Any attempt to launch from the torus outboard in such a speed domain would not be of use. Resulting deposition profiles then become even shallower. Hence, inboard launch is essential to allow for a suitable pellet fuelling performance. Taking an almost ideal launch configuration, as shown by the inset, deposition profiles are calculated (solid lines). Now, the high pressure induced drift of ablation cloud was taken into account. It becomes obvious taking advantage of the drift effect is crucial for DEMO core fuelling. For the $300 \mathrm{~m} / \mathrm{s}$ ITER reference case, a major fraction of pellet particles is deposited in the pedestal region. This would result in a significant fraction of poorly confined particles being quickly lost from plasma, creating a significant load on the pumping system. Such a large demand on the pumping capability would result in unreasonable requirements for the layout of the pumping system. Furthermore, the need to operate with higher total particle fuelling rates would also boost the fuel reservoir. However, restrictions to the fuel inventory form a critical bottleneck for DEMO. In order to stay within acceptable limits, imposed essentially for legislative approval, a solution of internal recycling, removing a significant amount of fuel from the fuel recovery and cleaning system [12] will be enforced.

In view of these circumstances, an improved fuelling efficiency with respect to the ITER reference case is most desirable. Increasing the pellet speed to $1000 \mathrm{~m} / \mathrm{s}$ results in a significant inboard relocation of the particles. An increase to $3000 \mathrm{~m} / \mathrm{s}$ further improves the situation, however only marginally. It is understood our first 
estimations do still lack the proper full treatment of the problem. For example, the pellet ablation and fuelling is not handled in a self-consistent way with respect to its impact on the target plasma. Such detailed modelling efforts are evidently scheduled for the progressing project, but it is also understood this is too ambiguous for a first explorative analysis. Nevertheless, our first explorative study already indicated it is potentially very rewarding to head for a solution in the speed range of about $1000 \mathrm{~m} / \mathrm{s}$ rather than sticking to the ITER solution [5]. Further improvements are expected to pay off only very marginally.

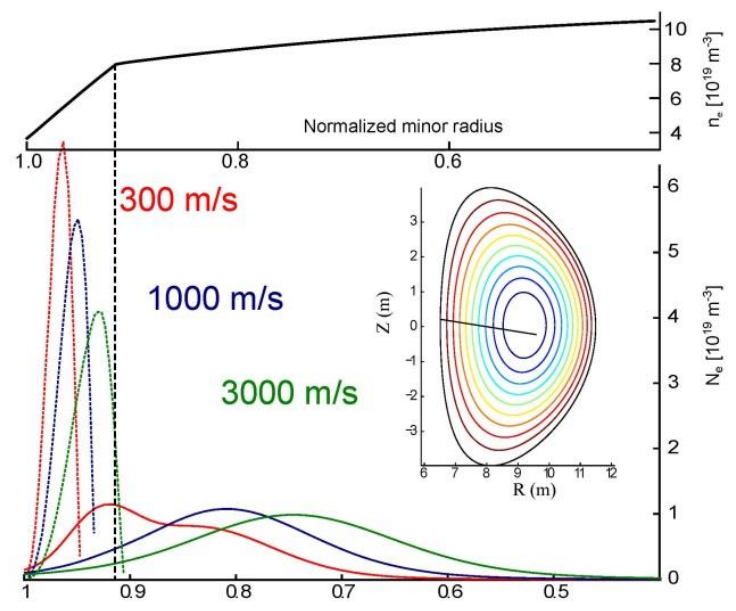

Fig. 2. Target density profile of the DEMO1noCD scenario (upper) and according ablation profiles (dashed lines) for a pellet size of $6 * 10^{21} \mathrm{D}$ at speed of $300 \mathrm{~m} / \mathrm{s}(\mathrm{red}), 1000 \mathrm{~m} / \mathrm{s}$ (blue) and $3000 \mathrm{~m} / \mathrm{s}$ (green). Assuming the launch geometry as shown by the insert, the accordingly colored deposition profiles (solid lines) result.

\section{Conventional pellet system components and their maturity for DEMO requirements}

It is now possible to take advantage of the exploration performed so far and set up a clear target for a first consideration of potential technical solutions. The potential of different options provided by different suitable matter injection techniques can be reviewed with respect to the estimated pellet parameter requirements. Although a broader view on any capable technique is envisaged, as a first step this consideration was focused on conventional pellet systems with a proven record in successful application in major fusion research devices. Taking a look at typical conventional systems, it is clear such a system is composed from three main components:

Pellet source delivers ice of the right size and consistency to the accelerating unit, forming the ice from an according gas mixture in the reservoir. This can be done as batch process or steady state.

Pellet accelerator section receives pellet from the source or cuts pellet with the required size from the ice ribbon provided and accelerates it to the pre-selected speed.

Pellet guiding system provides pellet transport to the desired launch position at the plasma boundary. In the past with injection primarily from the torus outboard side, this was often just a section of free flight granting a sufficient acceptance cone of the vessel. Now, with launching from the inboard prescribed, usually a tube guiding system is employed.

For such a serial process, the entire pellet launching systems performance and reliability is dictated by the product of the sub system values. Any weak component spoils the performance of the entire system. This applies also to the guiding system, a component often not designed with sufficient care and hence becoming the limiting subset of the system. Revisions of the guiding systems, especially close to or inside the torus vessel, then often turn out to require extensive efforts.

In the following, a short evaluation of the status of each of the three subsystems with respect to the current state of the art and the potential for DEMO is presented. Since in principle the components can be combined irrespective of their dedicated functions, at this stage any of the three components can be regarded as stand-alone sub systems.

The pellet source extruder technique can be considered to be well developed and on target to achieve the full capability required for reactor grade needs. Several steady state extrusion systems have proven their ability to deliver ice of sufficient quality and quantity reliably. For example, the screw extrusion system developed by the PELIN LLC for the JET HFPI [13] fulfils in routine operation any requirements with ease. Also, ORLN showed over decades their ability to design and build extrusion systems for a variety of launching systems. Recently, reliable steady state operation of the 1:5 ITER scale twin screw extruder was achieved [14]. Preparations for ITER's full prototype extruder are well under way. Thus, design and construction of a proper system for DEMO seems to be straight forward and possible from existing technology.

For pellet accelerators the same seems to hold true. There is a variety of different techniques to accelerate pellets up to speeds in the required range [15]. One major acceleration principle relies on momentum transfer from a streaming or expanding gas. In the case where the pellet dimension is smaller than the barrel diameter such a device is dubbed - Blower gun. Blower guns work reliably up to very high repetition rates of $100 \mathrm{~Hz}$ and beyond in the speed range $200-400 \mathrm{~m} / \mathrm{s}$. Higher velocities can be achieved by pneumatic gas guns where the pellet acts like a piston in the barrel driven by the expanding gas. Applying light gases like $\mathrm{H}$ or $\mathrm{He}$ as propellant, single stage guns can achieved velocities in excess of $1000 \mathrm{~m} / \mathrm{s}$ at repetition rates of several ten $\mathrm{Hz}$. For this gun type and speed range, already successful firing of $\mathrm{T}$ pellets has been demonstrated [16]. Pellet speeds in excess of $3000 \mathrm{~m} / \mathrm{s}$ can be realized by multi stage pneumatic guns, where high temperatures and pressures in the reservoir's final stage are generated by previous stage compressions. For this technique so far only quite low repetition rates have been demonstrated. Also, pellets might require embedding into a protective sabot due to the high thermal and stress load. Significant R\&D will be required to achieve DEMO relevant 
performance for this technique. Beside gas guns, mechanical devices relying on centrifugal force acceleration have been applied. For example, the ASDEX Upgrade (AUG) launcher [17] demonstrated velocities up to $1200 \mathrm{~m} / \mathrm{s}$ and repetition rates beyond 80 Hz. Taking the single stage gas gun or the centrifuge option, the $1000 \mathrm{~m} / \mathrm{s}$ speed range is well covered while $3000 \mathrm{~m} / \mathrm{s}$ would call for substantial R\&D efforts.

Pellet guiding systems as required by DEMO are likely to withstand around $1000 \mathrm{~m} / \mathrm{s}$, provided sufficient effort is applied to the design. For example, the AUG looping system, designed and tested for high speed transfer, is capable to launch into torus with up to $1040 \mathrm{~m} / \mathrm{s}$.

To conclude this first brief consideration: it seems pellet launch from the inboard side at about $1000 \mathrm{~m} / \mathrm{s}$ is possible in DEMO. It is apparent that most pellet transfer systems used to date suffer from too late or insufficient integration. It is therefore of primary importance that a request is made to the DEMO design team to include such a system from the inception stage, at least for the inner parts close to the wall inside the vacuum vessel.

\section{Integrating a high speed inboard launch guiding system into the actual DEMO design}

For the proposed lay out of the inner part of the DEMO pellet guiding tube system, we followed the same strategy as used for development of the AUG looping system. Notably, a very similar approach is currently also under way at JET, recognizing poor performance since 2008 caused by a bottleneck in the guiding system. It will be subject to a major revision until mid-2015. Rationalizing the LFS launch and going for sole inboard launch from the top of the machine it will come close to the DEMO design. This yields the opportunity for its use as a proper demonstration facility. The AUG looping was designed [18] based on results from Combs [15] on the pellet tensile strength. It is thought the pellet trajectory within a guiding system is a polygon track. The pellet is bouncing off the walls sufficiently protected by a cushion of ablated gas by the Leidenfrost effect until a critical perpendicular impact force is reached. Hence, the shape of the guiding system has to take into account all possible pellet trajectories and minimize the impact angle in order to maximize the transfer speed. This was done for the collimating funnel at the centrifuge exit where pellets are fed into the looping section. For this section a steady curvature of the track was stipulated in order to keep pellets well guided to the outer side of the tube. Any S-bending was strictly avoided since it causes pellets to cross the tube eventually impinging fatally on the opposite side.

Approximating the pellet motion by a steady sliding along the track contour, it becomes clear a higher curvature radius of the track contour favors higher transfer speeds. Ignoring any influence of the guiding tubes cross section assuming the pellet sliding along the tubes outer contour line of radius $R$, the critical maximum transfer speed $V_{c}$ can be estimated by balancing the resulting centrifugal forces on the pellet with the pellets critical yield strength $\sigma_{\mathrm{c}}$. For a cubic pellet with side length $L$ one gets:

$$
v_{\mathrm{c}}=\sqrt{\frac{\sigma_{c} R}{\rho L}}
$$

with $\rho$ the pellet density. Taking reasonable values for $\mathrm{D}$ ice at $10 \mathrm{~K}$ and the AUG system parameters, this estimation predicts a speed about 1.3 times the observed maximum transfer velocity. Possibly, this is due to an extrusion process not fully optimized hence resulting in a somewhat deteriorated ice quality - hinting that there is still headroom for further improvements in the AUG system. As a precaution, we took this into account and adopted the empirical "AUG calibrated" relation:

$$
v_{\mathrm{c}}\left[\frac{m}{\mathrm{~s}}\right]=1150 \sqrt{\frac{R[\mathrm{~m}]}{L[\mathrm{~mm}]}}
$$

First studies with the current EU DEMO model showed the possibility to integrate an appropriate guiding system with access from the vessel top, enabling inboard launches and obeying prescriptions as stated. Preliminary space reservations have already been made. However, for more detailed planning more considerations are inevitable. At present, there is a range of different transfer and launch solutions considered, a selection covering the range under investigation shown in figure 3.

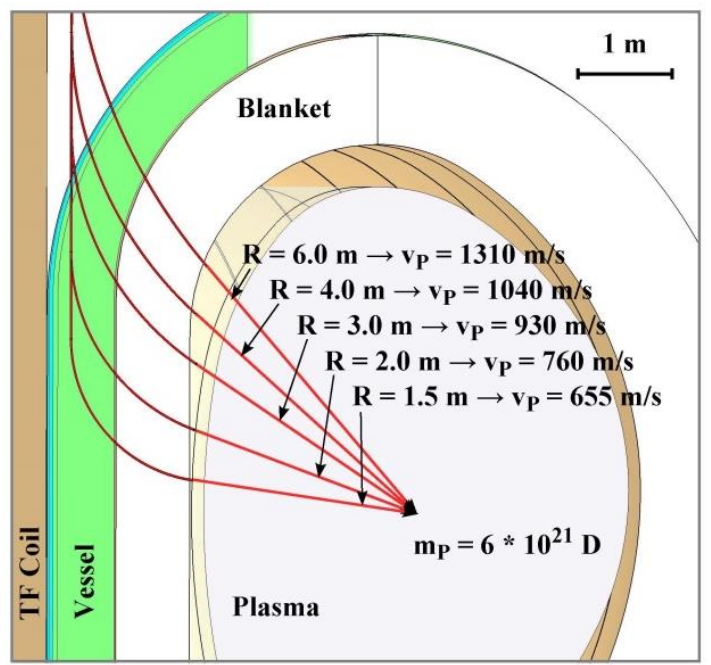

Fig. 3. Upper part of the DEMO cross section with plasma contour and innermost vessel and coil structures. Different possible solutions for the inboard track system are considered, the range represented by the 5 options displayed. For any option minimum bend radius and maximum expected transfer speed (assuming $6 * 10^{21} \mathrm{D}$ pellet mass) is indicated.

It displays the upper part of the DEMO cross section with the plasma contour and some of the innermost vessel and coil structures with several different options for the inboard track installation. For every option, the minimum bend radius and the according maximum transfer speed as estimated by the empirical relation and assuming a pellet mass of $6 * 10^{21} \mathrm{D}$ are indicated as well. Obviously, any option aiming to go for an almost ideal inboard launch close to the horizontal mid plane has to go through a quite narrow bend and will face 
significant speed restrictions. When relaxing these restrictions, some tilting angle between the final pellet trajectories directions into the plasma and the ideal horizontal solution becomes unavoidable. Hence, for the solution where the plasma drifts acts most favorably, a pellet speed restricted to about $700 \mathrm{~m} / \mathrm{s}$ can be expected. Higher launch velocities will be somewhat hampered by a declination between pellet flight path and the plasmoid drift direction expected along the magnetic field gradient. Evidently, well above $1000 \mathrm{~m} / \mathrm{s}$ according acceleration systems are needed as well. The best solution balancing both effects for a maximum overall fuelling performance is not yet known. It will require a detailed modelling taking into account pellet and plasma parameters but also the full complexity of the ablation and drift physics. Appropriate investigations are just about to begin, once they achieve a reliable state and result, the best option will be fixed within the DEMO design.

\section{Core fuelling technologies considered for the DEMO matter injection system}

Although the proposals presented above indicate a core particle fuelling system for DEMO based on conventional pellet technology can provide a sufficient solution, a yet broader approach considering alternative options is performed in parallel. In this assessment, all candidate techniques will be evaluated with respect to a requirement priority list still to be elaborated. A short list of matter injection techniques is presented. Still open to further extension it presently contains several approaches described in the following.

Conventional pellet injection as the prime candidate for the DEMO core fuelling system as considered above.

Microwave, laser ablation and rail gun pellet injection aiming to achieve even higher pellet velocities than achieved by the conventional launchers. These techniques rely on the rocket like repulsion by microwave or laser driven asymmetric heating of the pellet itself or an added pusher material [19]. Electromagnetic acceleration of a moving conductor pushing the pellet along the rails is used in rail guns [20].

Conventional gas puffing via conventional valves is used in all currently operated tokamaks as standard fuelling method, however usually limiting the accessible density. For core fuelling, due to edge density and the saturation effect predicted, conventional gas puffing most probably has to be disregarded but will nevertheless be analyzed.

Supersonic gas injection. To overcome limitations faced with a conventional gas injection system, a fast gas jet can be injected to establish a faster and highly directional gas flux. Usually, here the gas is injected from a supersonic Laval nozzle. Attempts are reported from several tokamaks as e.g. JT-60U [21] but also other fusion oriented devices.

Compact Tori Injection is expected to allow for launch speeds in the range of $100 \mathrm{~km} / \mathrm{s}$, making even central deposition accessible. These small toroidal confined plasma rings are usually compressed and accelerated by a coaxial rail gun. This concept has been seriously considered for ITER core fuelling purposes [4] but has been disregarded for several reasons: troublesome plasma access: too small particle inventory and impurity contamination from the rail gun. However, the technique deserves more careful consideration for DEMO.

Unmagnetized plasma jet injection. In order to allow for a higher density in the launched and injected structure compared to the compact tori, it was proposed to use an unmagnetized plasma jet alternatively. However, at present only design studies and simulations [22] seem to be available for this technique. Nevertheless, this approach will be considered.

\section{Summary}

A study is been initiated in the Tritium-Fuelling-Vacuum Project of the EUROFUSION DEMO programme working on the conceptual design of an optimized DEMO core particle fuelling system. The approach envisages an assessment of potential matter injection techniques finally aiming on design completion embedded in the DEMO fuel cycle concept. Considering as a first step conventional pellet launching it turns out inboard injection at about $1000 \mathrm{~m} / \mathrm{s}$ is technical feasible and seems to provide notable fuelling efficiency.

\section{Acknowledgments}

This work has been carried out within the framework of the EUROfusion Consortium and has received funding from the European Union's Horizon 2020 research and innovation programme under grant agreement number 633053. The views and opinions expressed herein do not necessarily reflect those of the European Commission.

\section{References}

[1] H. Zohm et al., Nucl. Fusion 53 (2013) 073019

[2] WORK PLAN FOR THE IMPLEMENTATION OF THE FUSION ROADMAP IN 2014-2018

https://www.euro-fusion.org/wpcms/wp-content/uploads/2013/01/JG12.356-web.pdf

[3] G.T.A. Huijsmans, Presentation at ITER PIS CDR (2014) ITER IDM: G4YED9

[4] R. Raman, Fusion Eng. Design 83 (2008) 1368

[5] L.R. Baylor et al., Nucl. Fusion 49 (2009) 085013

[6] P.T. Lang et al., Phys. Rev. Lett. 79 (1997) 1487

[7] W. Biel et al., this conference, I3.3

[8] B. Ploeckl, this conference, P3.035

[9] G.V. Pereverzev and Yu. Yushmanov, (1991) IPP Report 5/42

[10] R. Wenninger et al., Nucl. Fusion 54 (2014) 114003

[11] G.Cenacchi\&A.Taroni,1988, Rapporto ENEA RT/TIB (88)5

[12] C.Day and T. Giegerich, JoP: Fus. Eng. Des. 88 (2013) 616

[13] A. Geraud et al., Fusion Eng. Design 82 (2007) 2183

[14] S.K. Combs et al., Fusion Eng. Design 87 (2012) 634

[15] S. Combs, Rev. Sci. Instrum. 64 (1993) 1679

[16] P.W. Fisher et al., J. Vac. Sci. Technol. A7 (1989) 938

[17] C. Andelfinger et al., Rev. Sci. Instr. 64 (1993) 983

[18] A. Lorenz et al., Fusion Eng. Design 58-59 (2001) 325

[19] P.B. Parks and F.W. Perkins, Nucl. Fusion 46 (2006) 770

[20] M. Onozuka et al., Fusion Eng. Design 36 (1997) 451

[21] H. Takenaga et al., Nucl. Fusion 50 (2010) 115003

[22] W. Liu and S.C. Hsu, Nucl. Fusion 51 (2010) 073026 